\title{
Polaronic charge transport mechanism in DNA
}

\author{
D. Hennig ${ }^{\mathrm{a}}$, J. F. R. Archilla ${ }^{\mathrm{b}, 1}$, \\ ${ }^{a}$ Freie Universität Berlin, Fachbereich Physik, Institut für Theoretische Physik. \\ Arnimallee 14, 14195 Berlin, Germany \\ ${ }^{\mathrm{b}}$ Group of Nonlinear Physics, Departamento de Física Aplicada I \\ ETSI Informática, University of Sevilla, Avda Reina Mercedes, s/n. \\ 41012-Sevilla, Spain
}

\begin{abstract}
For the detailed understanding of the conduction mechanism in DNA we use models based on the concept of polaron and breather solutions. We describe how charge transport relies on the coupling of the charge carrying unit to the vibrational modes of DNA allowing for the formation of polaron-like localised states. The mobility of these localised states is discussed particularly in the presence of parametrical and structural disorder inherent to biomolecules. It is demonstrated that long-range coherent charge transport along the DNA structure is supported by mobile polaron and breather solutions suggesting that DNA seems suitable for the design of functional nanostructures as ingredients in molecular nanoelectronic devices.
\end{abstract}

Key words:

PACS: 87.-15.v,, 63.20.Kr, 63.20.Ry

\section{Introduction}

Electronic transport through DNA is crucial for its biological functions such as the repair mechanism after radiation damage and biosynthesis [1]. As proposed in [2] electron transport through DNA proceeds along a one-dimensional pathway constituted by the overlap between $\pi$-orbitals in neighboring base pairs along a strand. Recent measurements have indeed suggested that DNA forms an effective one-dimensional molecular wire [3]-[13] which offers promising applications in molecular electronics based on biomaterials [11],[12],[14].

$\overline{1}$ Corresponding author. E-mail:archilla@us.es

Preprint submitted to Recent Developments in Biopolymers July 5, 2005 
Opposed to that, it was reported that DNA is insulating [15] and for short oligomers built up from base pairs of the same type semiconductivity was observed [16]. This qualitative discrepancy, shared also by theoretical findings, instigated the still on-going debate whether DNA is conductive or not. In fact a theoretical investigation based on first principle calculations suggests the absence of dc-conductivity in DNA [17]. On the other hand there stand the studies using various assumptions for modeling the DNA structure which focus on different aspects such as the influence of aperiodicity, temperature driven fluctuations and aggregation effects on the energetic control of charge migration which have all come to the conclusion that DNA is a conductor [18]-[23].

The detailed understanding of the conduction mechanism of DNA remains still an unsolved task. Different attempts to model the charge transport of DNA were based on transport via coherent tunneling [2], classical diffusion under the conditions of temperature-driven fluctuations [20], incoherent phonon-assisted hopping [24],[25], variable range hopping between localized states [22] and charge carriages mediated by polarons [26]-[28] and solitons [29]. The impact of correlated disorder on the electronic conduction in DNA was studied recently in $[30]$.

Strongly backed by recent experimental results [31] theoretical considerations have been directed towards the idea that the constructive interplay between the charge carrying system and the vibrational degrees of freedom of DNA conspire to form mobile polarons which can establish coherent ET [20],[21]. Inspired by these observations we invoke for the theoretical description of charge transport in DNA the concept of polarons and breather solutions. The structure of the bent double helix of $\lambda$-DNA is modeled by a sterical network of oscillators in the frame of the base pair picture [32],[33],[34] taking into account deformations of the hydrogen bonds within a base pair and twist motions between adjacent base pairs. The electron motion is described by a tight-binding system. It is assumed that the electron motion is predominantly influenced by vibrational modes of the double helix. The nonlinear interaction between the electron and the vibrational modes causes the formation of polarons or electron-vibron breathers supporting charge localization and eventually transport.

We review the role played by static parametrical disorder and complex structural effects due to irregular deviations from the ordered helical shape of the double helix (reflected in random arrangements of the positions of the bases) with regard to the charge transport in DNA. Results concerning the charge transport in synthetic DNA polymers consisting of a single type of base pair are then represented. 


\section{Model Hamiltonian for polaron-like charge transfer along DNA}

DNA chains are quite flexible and exhibit structural fluctuations influencing the transport properties. In our DNA model we cover the basic features of the DNA double helix structure (abstracting from the detailed chemical structure) and consider the latter as a bent double-stranded system for which in the frame of a base pair picture the bases are treated as single nondeformable entities. The helicoidal structure of DNA is then conveniently described in a cylindrical reference system where each base pair possesses two degrees of freedom, namely a radial variable measuring the transversal displacements of the base pair (that is, deformations of the H-bond) and the angle with a reference axis in a plane perpendicular to the helix backbone which defines the twist of the helix [34]. In Fig. 1 we represent a sketch of the structure of the DNA model.

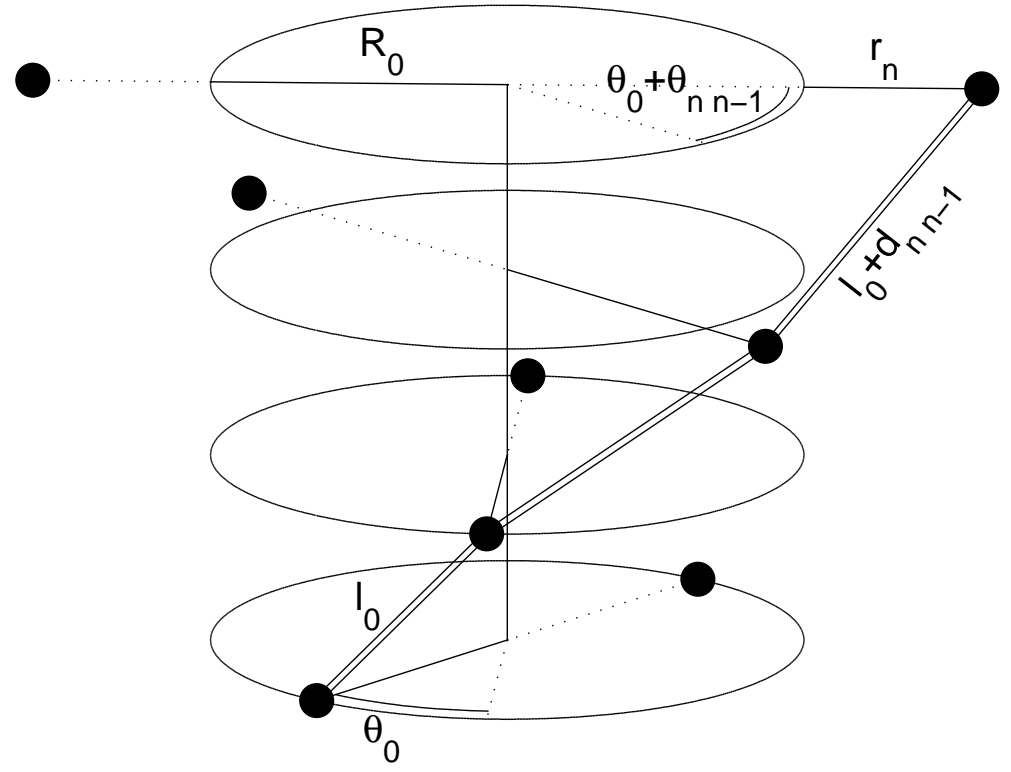

Fig. 1. Sketch of the helicoidal structure of the DNA model, the bases being represented by bullets. Geometrical parameters $R_{0}, \theta_{0}, l_{0}$ and the radial and angular variables $r_{n}$ and $\theta_{n n-1}$, respectively are indicated.

The charge migration process is influenced by the dominant vibrational modes of DNA stemming from transverse vibrations of the bases relative to each other, viz., the stretchings/compressions of the base pair distance within a base pair plane and the torsional variations of the helicoidal twist [20], [35]. The influence of other vibrational degrees of freedom (e.g. longitudinal acoustic phonons along the strands are significantly restrained by the stretch-proof sugar-phosphate chains in the backbone) can be discarded and the motion is restricted to the base pair planes [19]. Furthermore, the angular twist and radial vibrational motions evolve independently on two different time scales and 
can be regarded as decoupled degrees of freedom in the harmonic treatment of the normal mode vibrations of DNA [36].

The Hamiltonian for the electron migration along a strand in DNA consists of three parts

$$
H=H_{\mathrm{el}}+H_{\mathrm{rad}}+H_{\text {twist }}
$$

with $H_{\mathrm{el}}$ representing the part corresponding to the particle charge transport over the base pairs, $H_{\text {rad }}$ describes the dynamics of the H-bond vibrations and $H_{\text {twist }}$ models the dynamics of the relative twist angle between two consecutive base pairs. The electronic part is given by a tight-binding system

$$
H_{\mathrm{el}}=\sum_{n} E_{n}\left|c_{n}\right|^{2}-V_{n n-1}\left(c_{n}^{*} c_{n-1}+c_{n} c_{n-1}^{*}\right) .
$$

The index $n$ denotes the site of the $n$-th base on one of the two strands and $c_{n}$ determines the probability that the electron occupies this site. $E_{n}$ is the local electronic energy and $V_{n n-1}$ stands for the transfer matrix element which is responsible for the transport of the electron along the stacked base pairs.

The vibronic part $H_{\text {rad }}$ takes into account radial displacements of the base units from their equilibrium positions within the base pair plane connected with deformations of the hydrogen bonds linking two bases. The corresponding part of the Hamiltonian treating the bond vibrations classically and harmonically is given by

$$
H_{\mathrm{rad}}=\frac{1}{2} \sum_{n} M_{n}\left[\dot{r}_{n}^{2}+\Omega_{r}^{2} r_{n}^{2}\right]
$$

and the radial coordinate $r_{n}$ measures the deviation from the equilibrium length of a hydrogen bond along the line bridging two bases of a base pair, $\Omega_{r}$ is the frequency and $M_{n}$ is the reduced mass of a base pair.

The twist motion part reads as

$$
H_{\text {twist }}=\frac{1}{2} \sum_{n} J_{n}\left[\dot{\theta}_{n n-1}^{2}+\Omega_{\theta}^{2} \theta_{n n-1}^{2}\right]
$$

where $\theta_{n n-1}$ is the relative angle between two adjacent base pairs quantifying displacements from its equilibrium twist angle $\theta_{0}$ and $J_{n}$ is the reduced moment of inertia.

We emphasize that in the context of charge transport in DNA the nonlinear dynamics of large amplitude structural transitions are not of interest be- 
cause charge transport processes are thought to be mainly facilitated by soft fluctuational vibrational modes allowing the harmonic approximation of their dynamics.

The interaction between the electronic variable and the structure variables $r_{n}$ and $\theta_{n n-1}$ stems from the parameter dependence of the electronic parameters $E_{n}$ and $V_{n n-1}$. The diagonal term expressing the most efficient coupling is of the form

$$
E_{n}=E_{n}^{0}+k r_{n}
$$

and takes into account the modulation of the on-site electronic energy $E_{n}^{0}$ by the radial vibrations of the base pairs. In turn the actual charge occupation has its impact on the local radial distortion of the helix. As the transfer matrix elements $V_{n n-1}$ are concerned we assume that they depend on the three-dimensional distance between two consecutive bases along a strand in the following fashion

$$
V_{n n-1}=V_{0}\left(1-\alpha d_{n n-1}\right)
$$

The quantity $\alpha$ regulates how strong $V_{n n-1}$ is influenced by the distance and the latter is determined by

$$
\begin{aligned}
d_{n n-1} & =\left\{a^{2}+\left(R_{0}+r_{n}\right)^{2}+\left(R_{0}+r_{n-1}\right)^{2}\right. \\
& \left.-2\left(R_{0}+r_{n}\right)\left(R_{0}+r_{n-1}\right) \cos \left(\theta_{0}+\theta_{n n-1}\right)\right\}^{1 / 2}-l_{0},
\end{aligned}
$$

with

$$
l_{0}=\sqrt{a^{2}+4 R_{0}^{2} \sin ^{2}\left(\theta_{0} / 2\right)} .
$$

Expanding the expression (7) up to first order around the equilibrium positions gives

$$
d_{n n-1} \simeq \frac{R_{0}}{l_{0}}\left[\left(1-\cos \theta_{0}\right)\left(r_{n}+r_{n-1}\right)+\sin \theta_{0} R_{0} \theta_{n n-1}\right]
$$

Realistic parameters for DNA molecules are given by [34],[35]: $a=3.4 \AA$, $R_{0} \approx 10 \AA, \theta_{0}=36^{\circ}, J=4.982 \times 10^{-45} \mathrm{~kg} \mathrm{~m}^{2}, \Omega_{\theta}=[0.526-0.744] \times 10^{12} \mathrm{~s}^{-1}$, $\Omega_{r}=6.252 \times 10^{12} \mathrm{~s}^{-1}, V_{0} \simeq 0.1 \mathrm{eV}$ and $M=4.982 \times 10^{-25} \mathrm{~kg}$.

We scale the time according to $t \rightarrow \Omega_{r} t$ and introduce the dimensionless quantities: 


$$
\begin{aligned}
& \tilde{r}_{n}=\sqrt{\frac{M \Omega_{r}^{2}}{V_{0}}} r_{n}, \quad \tilde{k}_{n}=\frac{k_{n}}{\sqrt{M \Omega_{r}^{2} V_{0}}}, \quad \tilde{E}_{n}^{0}=\frac{E_{n}^{0}}{V_{0}} \\
& \tilde{\Omega}=\frac{\Omega_{\theta}}{\Omega_{r}}, \quad \tilde{V}=\frac{V_{0}}{J \Omega_{r}^{2}}, \quad \tilde{\alpha}=\sqrt{\frac{V_{0}}{M \Omega_{r}^{2}}} \alpha, \quad \tilde{R}_{0}=\sqrt{\frac{M \Omega_{r}^{2}}{V_{0}}} R_{0} .
\end{aligned}
$$

Subsequently we omit the tildes.

With the use of the expression (9) the equations of motion derived from the Hamiltonian (2)-(4) read as

$$
\begin{aligned}
\mathrm{i} \tau \dot{c}_{n} & =\left(E_{n}^{0}+k r_{n}\right) c_{n} \\
& -\left(1-\alpha d_{n+1, n}\right) c_{n+1}-\left(1-\alpha d_{n n-1}\right) c_{n-1} \\
\ddot{r}_{n} & =-r_{n}-k\left|c_{n}\right|^{2}-\alpha \frac{R_{0}}{l_{0}}\left(1-\cos \theta_{0}\right) \\
& \times\left\{\left[c_{n+1}^{*} c_{n}+c_{n+1} c_{n}^{*}\right]+\left[c_{n}^{*} c_{n-1}+c_{n} c_{n-1}^{*}\right]\right\} \\
\ddot{\theta}_{n n-1} & =-\Omega^{2} \theta_{n n-1}-\alpha V \frac{R_{0}^{2}}{l_{0}} \sin \theta_{0}\left[c_{n}^{*} c_{n-1}+c_{n} c_{n-1}^{*}\right],
\end{aligned}
$$

and the quantity $\tau=\hbar \Omega_{r} / V_{0}$ appearing in Eq. (12) determines the time scale separation between the fast electron motion and the slow bond vibrations. We remark that in the limit case of $\alpha=0$ and constant $E_{n}^{0}=E_{0}$ the set of coupled equations represents the Holstein system widely used in studies of polaron dynamics in one-dimensional lattices [37]. Furthermore in the linear limit case emerging for $\alpha=k=0$ the Anderson model is obtained for random $E_{n}^{0}[38],[39]$. We remark that the underlying model is suitable for the study of charge transport in DNA in a vacuum when, in particular, the damping influence of the biological environs on the structural dynamics can be discarded. The values of the scaled parameters are given by $\Omega^{2}=[0.709-1.417] \times 10^{-2}$, $V=0.0823, R_{0}=34.862$ and $l_{0}=24.590$. In our model study we treat the electron-mode coupling strengths $k$ and $\alpha$ as adjustable parameters and adopt the values of these free parameters such that not too strong deformations of the helix result which is essential for our used harmonic treatment of the dynamics of the structural coordinates. Afterwards we study synthetic DNA molecules consisting of a single type of base pairs for which we are able to compute reliable values of the coupling strengths with quantum chemical methods (see further below).

\section{Localized polaron-like states}

Caused by the nonlinear interplay between the electronic and the vibrational degrees of freedom of the helix the formation of polaronic electron-vibration 
compounds is possible [20],[21],[24],[26],[40], [41], [42]. Being interested in the simulation of a nonlinear charge transport mechanism in DNA we embark as a first step on the construction of localized stationary solutions of the coupled system (12)-(14). Exploiting the fact that the adiabaticity parameter $\tau$ is small, i.e. the fast charge transport along the base stacks and the slow bond vibrations and the even slower rotational twist motions evolve on distinct time scales, the inertia in Eqs. (13) and (14) are negligible. Therefore, one can solve in the adiabatic limit the resulting static equations which results in the instantaneous displacements

$$
\begin{aligned}
r_{n} & =-k\left|c_{n}\right|^{2}-\alpha \frac{R_{0}}{l_{0}}\left(1-\cos \theta_{0}\right) \\
& \times\left\{\left[c_{n+1}^{*} c_{n}+c_{n+1} c_{n}^{*}\right]+\left[c_{n}^{*} c_{n-1}+c_{n} c_{n-1}^{*}\right]\right\}, \\
\theta_{n n-1} & =-\frac{\alpha V}{\Omega^{2}} \frac{R_{0}^{2}}{l_{0}} \sin \theta_{0}\left[c_{n}^{*} c_{n-1}+c_{n} c_{n-1}^{*}\right] .
\end{aligned}
$$

Upon insertion of (15) and (16) into Eq. (12) one obtains a nonlinear discrete Schrödinger equation for the electronic amplitude

$$
\begin{aligned}
\mathrm{i} \tau \dot{c}_{n} & =\left[E_{n}^{0}-k^{2}\left|c_{n}\right|^{2}-k \alpha \frac{R_{0}}{l_{0}}\left(1-\cos \theta_{0}\right)\right. \\
& \left.\times\left\{\left[c_{n+1}^{*} c_{n}+c_{n+1} c_{n}^{*}\right]+\left[c_{n}^{*} c_{n-1}+c_{n} c_{n-1}^{*}\right]\right\}\right] c_{n} \\
& -\left(1+b_{n+1 n}\right) c_{n+1}-\left(1+b_{n n-1}\right) c_{n-1}
\end{aligned}
$$

with

$$
\begin{aligned}
b_{n n-1} & =\alpha\left(\frac{R_{0}}{l_{0}}\right)^{2}\left\{( 1 - \operatorname { c o s } \theta _ { 0 } ) \left[k \frac{l_{0}}{R_{0}}\left[\left|c_{n}\right|^{2}+\left|c_{n-1}\right|^{2}\right]\right.\right. \\
& +\alpha\left(1-\cos \theta_{0}\right)\left(\left[c_{n+1}^{*} c_{n}+c_{n+1} c_{n}^{*}\right]\right. \\
& \left.\left.+2\left[c_{n}^{*} c_{n-1}+c_{n} c_{n-1}^{*}\right]+\left[c_{n-1}^{*} c_{n-2}+c_{n-1} c_{n-2}^{*}\right]\right)\right] \\
& \left.+\frac{\alpha V}{\Omega^{2}} R_{0}^{2} \sin ^{2} \theta_{0}\left[c_{n}^{*} c_{n-1}+c_{n} c_{n} c_{n-1}^{*}\right]\right\}
\end{aligned}
$$

In order to search for stationary localized solutions (polaron states) we substitute $c_{n}=\Phi_{n} \exp [-i E t / \tau]$ in Eq. (17) and obtain the difference system

$$
\begin{aligned}
E \Phi_{n} & =\left[E_{n}^{0}-k^{2}\left|\Phi_{n}\right|^{2}-k \alpha \frac{R_{0}}{l_{0}}\left(1-\cos \theta_{0}\right)\right. \\
& \left.\times\left\{\left[\Phi_{n+1}^{*} \Phi_{n}+\Phi_{n+1} \Phi_{n}^{*}\right]+\left[\Phi_{n}^{*} \Phi_{n-1}+\Phi_{n} \Phi_{n-1}^{*}\right]\right\}\right] \Phi_{n} \\
& -\left(1+B_{n+1 n}\right) \Phi_{n+1}-\left(1+B_{n n-1}\right) \Phi_{n-1},
\end{aligned}
$$


where $B_{n n-1}$ is given by

$$
\begin{aligned}
B_{n n-1} & =\alpha\left(\frac{R_{0}}{l_{0}}\right)^{2}\left\{( 1 - \operatorname { c o s } \theta _ { 0 } ) \left[k \frac{l_{0}}{R_{0}}\left[\left|\Phi_{n}\right|^{2}+\left|\Phi_{n-1}\right|^{2}\right]\right.\right. \\
& +\alpha\left(1-\cos \theta_{0}\right)\left(\left[\Phi_{n+1}^{*} \Phi_{n}+\Phi_{n+1} \Phi_{n}^{*}\right]\right. \\
& \left.\left.+2\left[\Phi_{n}^{*} \Phi_{n-1}+\Phi_{n} \Phi_{n-1}^{*}\right]+\left[\Phi_{n-1}^{*} \Phi_{n-2}+\Phi_{n-1} \Phi_{n-2}^{*}\right]\right)\right] \\
& \left.+\frac{\alpha V}{\Omega^{2}} R_{0}^{2} \sin ^{2} \theta_{0}\left[\Phi_{n}^{*} \Phi_{n-1}+\Phi_{n} \Phi_{n-1}^{*}\right]\right\}
\end{aligned}
$$

The ground state of the system (19) is computed with the numerical map method outlined in [43],[44].

In Fig. 2 we depict typical patterns of polaron states. First we discuss the ordered (periodic) case arising e.g. for synthetically produced DNA molecules consisting of a single type of base pairs (e.g. poly $(G)$-poly $(C)$ DNA polymers) surrounded by vacuum which provides realistic ideal conditions for the consideration of DNA conductivity in experimental and theoretical studies [16]. The electronic contribution is shown in Fig. $2(a)$. In the ordered case the electronic wave function is localized at the central lattice site and the amplitudes decay monotonically and exponentially with growing distance from this central site. We remark that the spatial extension of the polaron decreases with increased coupling strength $k$ and there is a smooth transition from large to small polarons. Analogously, enlarging the off-diagonal coupling strength $\alpha$ leads also to enhanced degree of localization. The size of the polarons plays a crucial role for their mobility in the sense that for large up to medium polarons coherent motion can be activated whereas small polarons are immobile due to their pinning to the discrete lattice [45],[46]. The associated patterns of the static radial and angular displacements of the bases are shown in Fig. $2(b)$ and $2(c)$, respectively. Like the electronic wave function the radial and angular displacements are exponentially localized at the central lattice site (base pair). Notice that due to the overall minus sign of the radial and angular excitation patterns the H-bridges get compressed while the helix experiences a local unwinding around the occupation peak of the localized electron.

For a more realistic study we take static diagonal disorder in the on-site electronic energy $E_{n}^{0}$ into account. The random values caused for example by the inhomogeneous broadening of the sites of distinct base pairs with different energies were simulated by random potentials $[-\Delta E, \Delta E]$ with mean value $\bar{E}=0$ and different mean standard deviations $\Delta E$. (Note that any $E_{0} c_{n}$ term on the r.h.s. of Eq. (12) with constant $E_{0}$ can be eliminated by a gauge transformation $c_{n} \rightarrow \exp \left(-\mathrm{i} E_{0} t / \tau\right) c_{n}$.)

Generally the localized excitation patterns do not change qualitatively when 


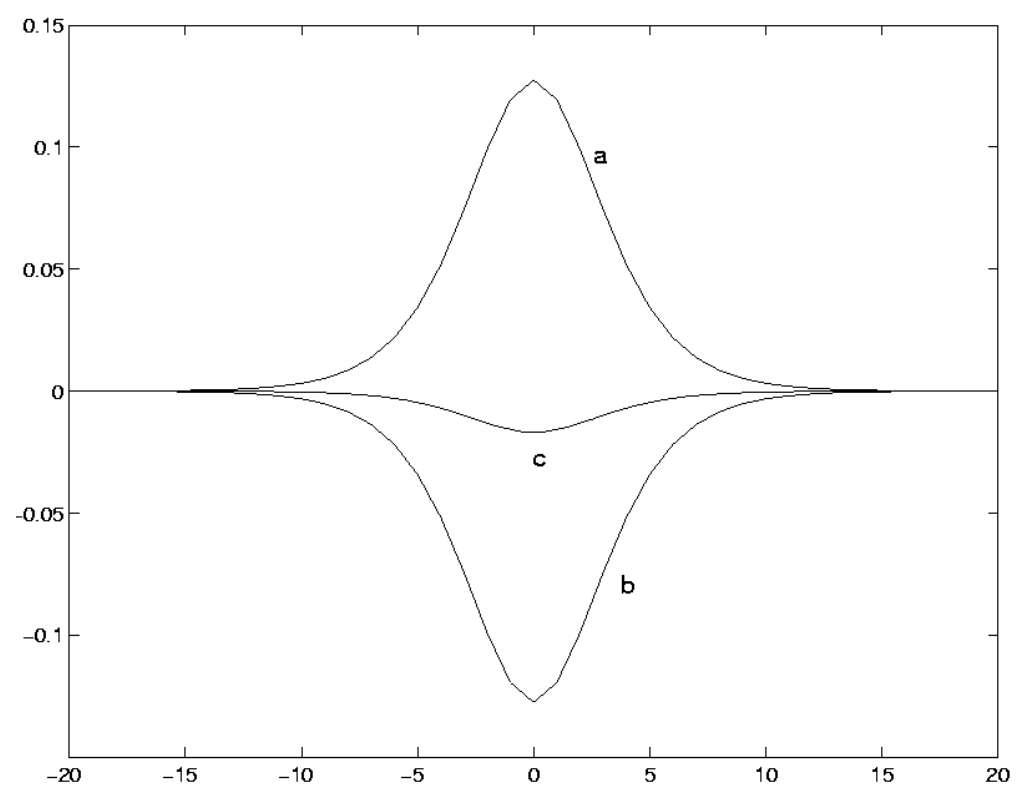

Fig. 2. Profiles of the stationary polaron states for the ordered (periodic) case.

(a) The electronic amplitudes $\left|\Phi_{n}\right|^{2}$.

(b) The static radial displacements $r_{n}$.

(c) The static angular twists $\theta_{n}$.

diagonal disorder is taken into account. Compared with the ordered case one merely observes a shift of the excitation peak away from the central lattice site depending on the respective realization of the random potential. However, since in the presence of diagonal disorder the translational invariance of the lattice system is broken the localized amplitude pattern is no longer reflection symmetric with respect to a distinguished lattice site as opposed to the corresponding symmetric localized state of the ordered system. Moreover we find that the combined effect of the two localization mechanisms, viz. nonlinear polaron and linear Anderson-mode formation respectively, leads to enhancement of the degree of localization compared to the case when only one of the two mechanisms acts.

Furthermore, real DNA molecules exhibit random structural imperfections of their double helix caused e.g. by the random base pair sequence and the solvent conditions DNA that may leave the involved helix structures in irregularly distorted shapes. Accordingly, the structural disorder is incorporated in our model by randomly distributed equilibrium positions of the bases so that the resulting irregular helical DNA matrix deviates from the perfectly regular helix structure.

To be precise, we consider randomly distributed torsional coordinates $\theta_{n n-1}$ and radii $r_{n}$. Since the positions (actually, the distances) govern the value of the corresponding transfer matrix elements $V_{n n-1}=V_{0}\left(1-\alpha d_{n n-1}\right)$ nondi- 
agonal disorder is induced in the latter at the same time. Random transfer integrals along the helix were used to represent random base pair sequences for which the charge transport along DNA was discussed recently in a model relying on localized electron states [19].

\section{Charge transport established by mobile polarons}

We study now the charge transport supported by mobile polarons propagating along DNA. To activate polaron motion an established method is provided by proper stimulation of localized internal normal modes of certain shape and frequency which are also called pinning modes [47]. In this way kinetic energy is injected into the system in the form of an initial kick of the momentum variables of the vibrational degrees of freedom. Results regarding activation of polaron motion in the ordered case are presented in Fig. 3. We integrated the set of coupled nonlinear equations (12)-(14) with a fourth-order Runge-Kutta method and the accuracy of the computation was checked through monitoring the conservation of the total energy as well as the norm $\sum_{n}\left|c_{n}(t)\right|^{2}=1$. The applied kicking strength is $\lambda_{r}=0.02$ corresponding to an amount of radial kinetic energy of the order of $200 \mathrm{meV}$. Interestingly, we found that there exists no angular pinning mode component $\xi_{\theta}$ (see further below) so that alone the excitation of the radial component $\xi_{r}$ produces long-lived stable polaron and breather motions. Hence, our findings suggest that higher frequency radial fluctuational modes of DNA instigate the propagation of localized structures along DNA rather than low frequency twist fluctuational modes. The Fig. $3(a)$ demonstrates that the electronic component of the polaron moves with uniform velocity along the lattice maintaining its localized shape throughout the journey. In the same manner the radial polaron part travels as a localized wave along the strand so that the electron propagation is accompanied by a local and temporal contraction of the radii. However, the angular static polaron component splits up into two parts when the radial pinning mode is initially imposed. At the starting position there remains a standing breather of comparatively large amplitudes which reverse periodically their sign in the course of time. Thus the helix experiences alternately a local winding and unwinding around its central base pair. In addition a second breather of relatively small and exclusively negative amplitude propagates in unison with the electronic and radial polaron components. The result is that the region of the helix which is traversed by the electron gets locally untwisted. As the quantitative assessment of the charge transport is concerned we note that the breather travels across seventy sites (base pairs) in a thousand (dimensionless) time units meaning that physically the charge conduction across the base pairs of DNA over a distance of $238 \AA$ takes $\backsim 0.16 \mathrm{~ns}$ leading to a higher transport rate than a typical one found for protein conductivity [19]. This underlines 
the status ascribed to DNA molecules as promising candidates establishing fast and efficient charge transport in (albeit only theorized yet) molecular electronical engineering utilizing them as molecular wires.

In the presence of diagonal static disorder, that is when the on-site electronic energies $E_{n}^{0}$ are represented by random numbers distributed in the interval $\left|E_{n}^{0}\right|<\Delta E$ with width $\Delta E$, we find that mobile breathers exist up to a critical degree of disorder $\Delta E_{\text {crit }}$ and ET persists. (Let us stress that it was indeed experimentally found that the random sequence $\lambda-\mathrm{DNA}$ is an electrical conductor [8].) Beyond this critical value continuous propagation of the breathers along the lattice is prevented. More precisely, while for a relatively small amount of disorder $\Delta E=0.05$ the electronic breather (together with its radial and torsional counterparts) is able to travel uniformly along the lattice for a ten times increased $\Delta E=0.5$ the breather motion is restricted to a neighborhood of the initially excited position around which it wanders in an oscillatory manner. The temporal evolution of the electron breather center are shown in Fig. 4.

Concerning the impact of structural disorder we considered random distributions of the base pair spacings $r_{n}-r_{0} \in[-\Delta, \Delta]$ and the twist angles $\theta_{n}-\theta_{0} \in[-\Delta, \Delta]$ with different mean standard deviations $\Delta$.

For representative results we averaged over several realizations of structural disorder. The results for the temporal evolution of the electron breather center are depicted in Fig. 5. We conclude that structural disorder does not significantly affect the mobility of the breathers. For growing degree of disorder merely the propagation velocity gets reduced. Even in the case of fairly strong disorder $\Delta=10 \%$ (dashed line) the breather is still able to propagate steadily.

We studied also the polaron mobility under the impact of combined static diagonal and structural disorder and found that the strong pinning effect generated by static diagonal disorder prevails over the more harmless and merely velocity-reducing influence of structural irregularities. Particularly the last result differs from the opposing finding reported in [48] suggesting that hopping conductivity in proteins and DNA seems to be influenced first of all by the molecular conformations and not by their sequences.

\section{Charge transport in poly(dA)-poly(dT) and poly(dG)-poly(dc) DNA polymers}

In this section we theoretically describe the charge transport in synthetic DNA polymers built up from single types of base pairs on the basis of the model 

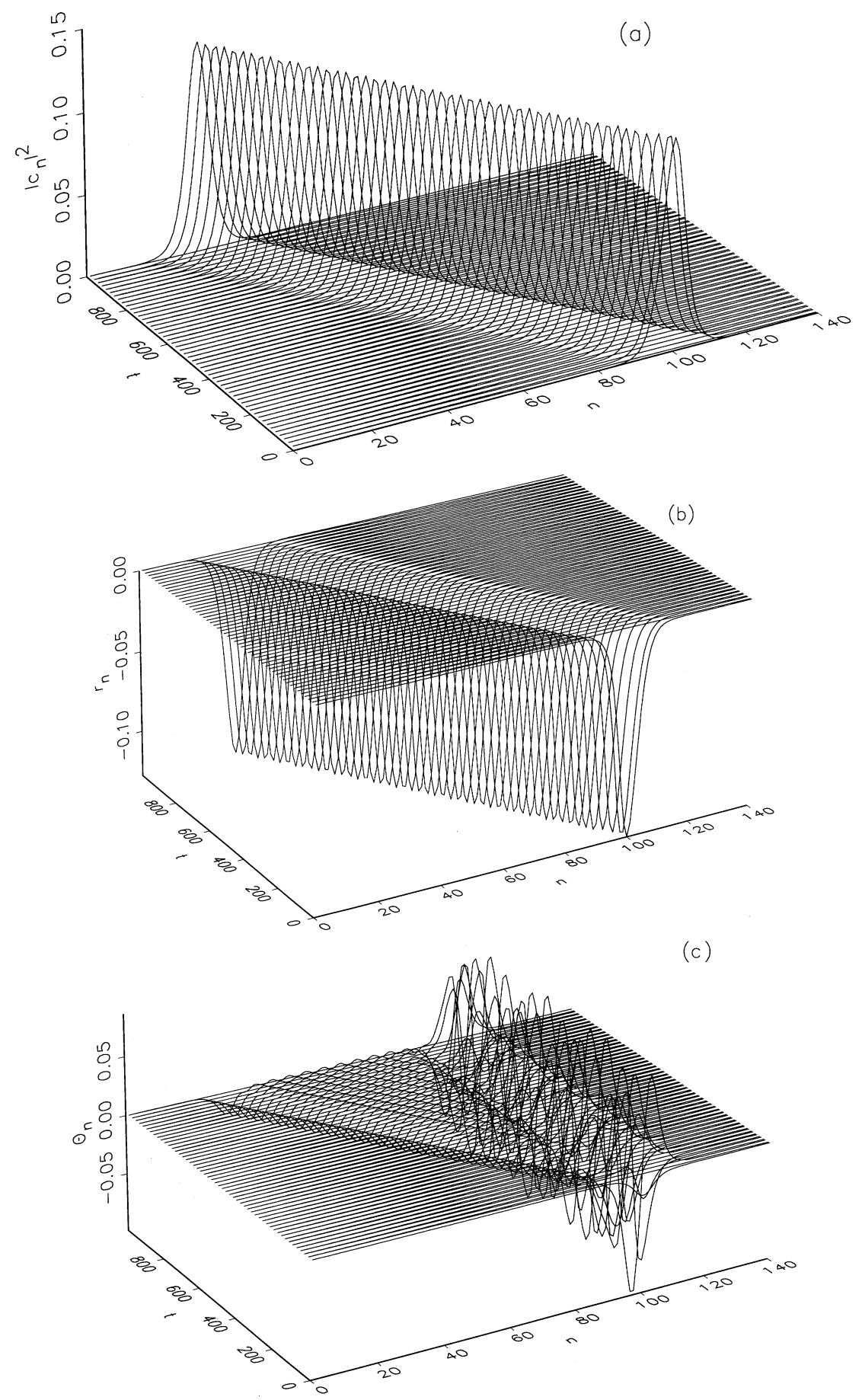

Fig. 3. Breather motion along the DNA in the ordered case. Motion is initiated by an initial kick of the velocities $\left\{\dot{r}_{n}\right\}$ directed along the corresponding pinning mode component. The kick amplitude is $\lambda_{r}=0.25$. (a) Uniformly moving electronic breather.(b) The associated moving breather in the radial displacements. (c) The angular breather contribution consisting of a small amplitude breather pinned at the starting site and a relatively large amplitude moving breather component propagating in alliance with the electron and radial breathers. 


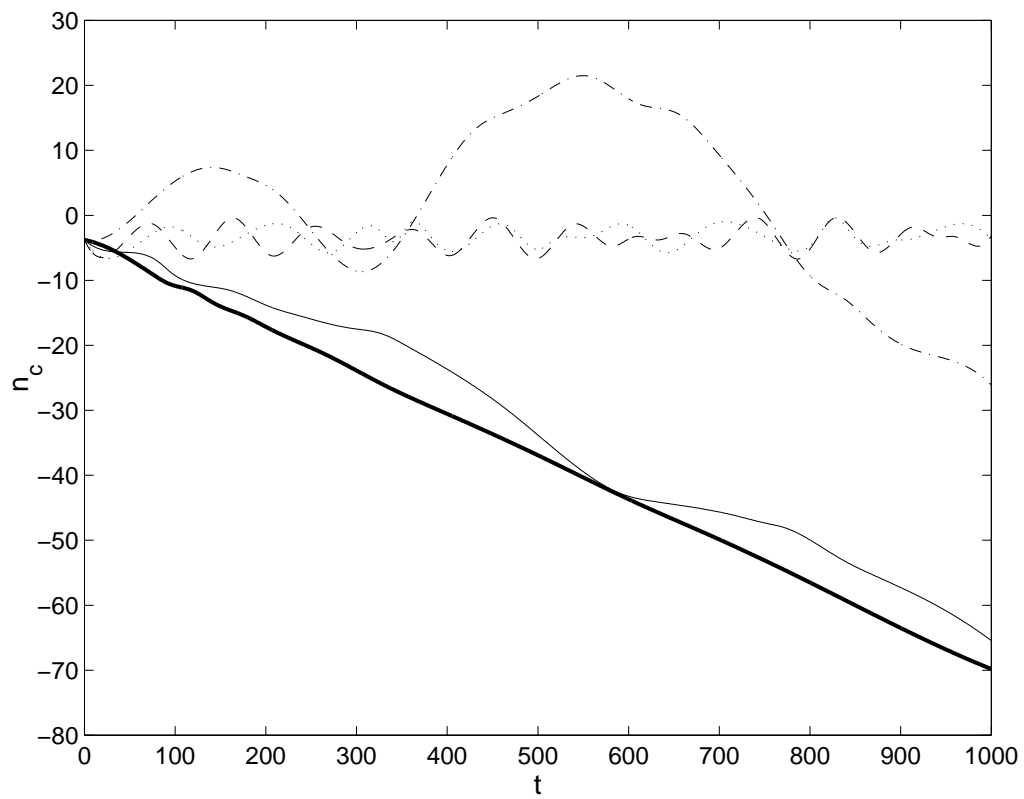

Fig. 4. Diagonal disorder. The position of the center of the electron breather as a function of time and for different amounts of disorder. Full line (Ordered case), short dashed line $(\Delta E=0.025)$, dashed-dotted line $(\Delta E=0.050)$, dotted line $(\Delta E=0.100)$ and long dashed line $(\Delta E=0.500)$.

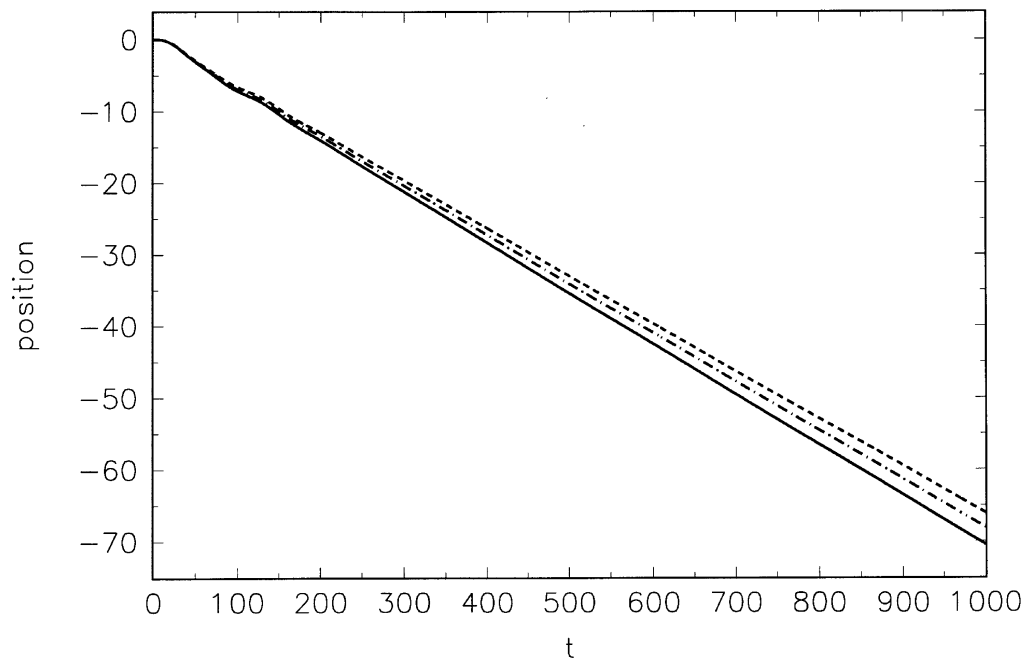

Fig. 5. Structural disorder and random double helix structure. The temporal behavior of the position of the electron breather center for different degrees of randomness in the radii $r_{n}$ and angles $\theta_{n}$. Assignment of the line types to the increasing mean standard deviations ranging up to $10 \%$ of the respective mean value: Full line (1\%), dashed dotted line (5\%) and dashed line (10\%).

introduced above.

Concerning the interaction between the electronic and the vibrational degrees of freedom the quantum-chemical computation of the geometry dependence 
of the electronic parameters $E_{n}$ and $V_{n n-1}$ reveals their most significant modulation originates from radial distortions of the helix which are related with hydrogen and covalent bond deformations, respectively. However, the influence of small angle deformations on the values of the electronic parameters can be discarded. Hence the corresponding twist part can be omitted of the Hamiltonian (4) in a forthcoming study.

Furthermore, distinct from the previous studies we use for our computations credible values for the electron-mode coupling strengths $k$ and $\alpha$ as a result of quantum-chemical computational procedure described in Refs. [53,54]. To this end it has been performed quantum-chemical calculations on symmetrical homodimers consisting of two nucleoside Watson-Crick base pairs (adenosinethymidine (AT) and guanosine-cytidine (GC) base pair steps (BPS)) stacked over each other to mimic the conventional A-DNA and B-DNA conformations [49]. Taking into account DNA backbone at least in form of intact sugar moieties, instead of substituting it by protons or methyl groups, is necessary for the consistency of the calculations [50] and to correctly describe charge transfer through DNA duplexes [51]. E.B. Starikov [53] has used semiempirical all-valence-electron PM3 Hamiltonian [52] within the MOPAC7 version of CI (configuration interaction) approximation. PM3-CI method is chosen, but not an ab initio one, since

a) The molecular fragments involved are very large;

b) Similar ab initio calculations even for smaller segments experience difficulties with SCF convergence [55];

c) The work [50] used similar semiempirical Hamiltonian (AM1) for analogous molecular fragments;

d) PM3-CI approximation is good at describing excited states of nucleoside base pair steps [53];

e) CI approximation is indispensable when charged states of nucleic acid bases are considered using semiempirical quantum chemistry (cf., e.g., [56] and references therein).

Here the effects of small, but non-negligible (up to 0.1 ), radial stretching and compressing of Watson-Crick hydrogen bonds (WC H-bonds) on the MO energies of the BPS under study have been estimated. Specifically, In the above sense the equilibrium lengths of the $\mathrm{WC} \mathrm{H}$-bonds are perturbed in only one of the base pairs in all the BPS involved and the resulting changes in the energies of the highest occupied molecular orbital and of the occupied molecular orbital next to the highest one (HOMO and HOMO-1, respectively), as compared with the equilibrium values of these energies, are monitored. According to the Koopmans theorem well known in quantum chemistry (cf., 
e.g., [55] and references therein), the HOMO energy is approximately equal to molecular ionisation potential and in the tight-binding approximation could be viewed as the site energy, whereas the difference between the HOMO and HOMO-1 energies is approximately twice the hopping integral. With these considerations, the $k$ and $\alpha$ parameters of our model can be estimated by calculating linear regression of the corresponding site energy and hopping integral changes, respectively, onto WC H-bond distance perturbations. A very tight linear correlation between the former and the latter ones is achieved. Interestingly, there is not any correlation between the tight-binding Hamiltonian parameter changes and BPS twist angle/helical pitch perturbations ('helical pitch' is the distance $a$ in Eq. (8)). The perturbations in the two latter parameters are also relatively small (up to 10 degrees and 0.1 , respectively). For the coupling parameters of the poly(dA)-poly(dT) DNA polymer the valued obtained are: $k=0.0778917 \mathrm{eV} / \AA$ and $\alpha=0.053835 \AA^{-1}$. The corresponding values for the poly $(\mathrm{dG})$-poly $(\mathrm{dC})$ DNA polymer are determined as $k=-0.090325 \mathrm{eV} / \AA$ and $\alpha=0.383333 \AA^{-1}$.

Like in the previous study we construct stationary localized (polaronic) electronvibration compounds with the help of the nonlinear map approach. In Fig. 6 we depict the profiles of the (standing) polaron states for the poly $(\mathrm{dA})$-poly $(\mathrm{dT})$ and the poly $(\mathrm{dG})$-poly $(\mathrm{dC})$ DNA polymer, respectively. In both cases the polarons are of fairly large extension (width). Regardless of the DNA polymer type the electronic wave function is localized at lattice site and the envelope of the amplitudes decays monotonically and exponentially with growing distance from this central site (base pair). However, the electronic wave function of the poly $(\mathrm{dG})$-poly $(\mathrm{dC})$ DNA polymer is stronger localized than the one of its poly $(\mathrm{dA})$-poly $(\mathrm{dT})$ counterpart. Accordingly, the attributed radial displacement patterns are exponentially localized at the central lattice site. Concerning the resulting static radial helix deformations we find that there is a drastic difference between the poly $(\mathrm{dA})-\operatorname{poly}(\mathrm{dT})$ and the poly $(\mathrm{dG})$-poly $(\mathrm{dC})$ DNA polymers. In the former case the overall non-positive radial amplitudes imply that the H-bridges experience contractions. In contrast, in the latter case the H-bridges get stretched. Nevertheless, these deformations are rather weak, i.e. on the order of $1.510^{-3} \AA$.

As the ET is concerned polaron motion is activated with the use of the discrete gradient method [57]. With this method we obtain suitable initial perturbations of the momentum coordinates $p_{n}^{r}$ which initiate coherent motion of the polaron compound.

We consider first the case of the poly $(\mathrm{dG})$-poly $(\mathrm{dC})$ DNA polymer. Interestingly, only the electronic component sets off to move directionally along the lattice whereas the vibrational amplitude pattern remains at the initial position. Hence, long-range ET is achievable. Contrary to that we found that the poly(dA)-poly(dT) DNA polymer does not exhibit such good charge mobil- 

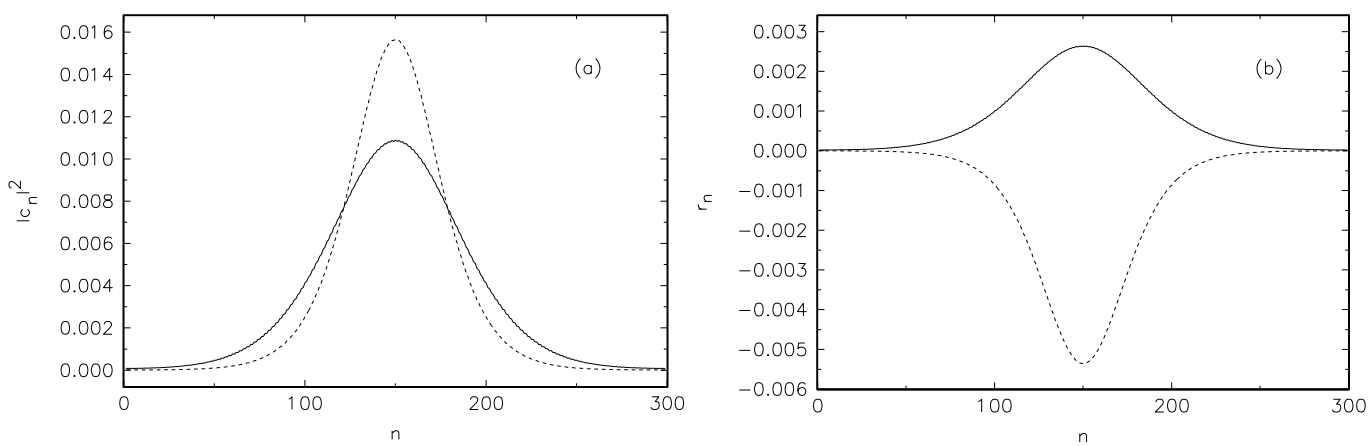

Fig. 6. The spatial pattern of the polaronic electron-vibration compound. (a) The electronic part. Full (dashed) line: poly $(\mathrm{dA})$-poly $(\mathrm{dT})$ (poly $(\mathrm{dG})$-poly $(\mathrm{dC})$ ) DNA polymer. (b) Radial deformation pattern. Assignment of line types as in (a).

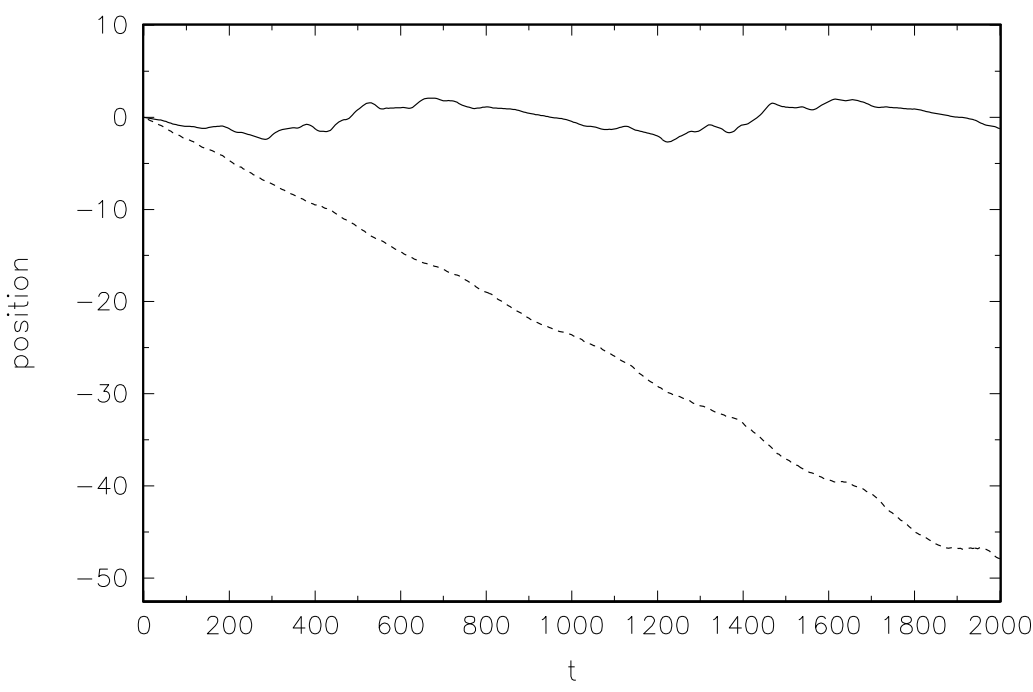

Fig. 7. Time evolution of the first momentum of the electronic occupation probability. Full (dashed) line: poly (dA)-poly(dT) (poly(dG)-poly(dC)) DNA polymer.

ity as its poly $(\mathrm{dG})-$ poly $(\mathrm{dC})$ counterpart. The different propagation scenarios are properly illustrated using the time evolution of the first momentum of the electronic occupation probability defined as $\bar{n}(t)=\sum_{n} n\left|c_{n}(t)\right|^{2}$. One can clearly observe that in the $(\mathrm{dG})-(\mathrm{dC})$ case electron propagation proceeds uninhibitedly with uniform velocity (see Fig. 7). In comparison the (dA)-(dT) electron travels with smaller velocity and after having traversed eight lattice sites its motion stops so that the electron becomes trapped eventually.

In conclusion, we have found that conductivity in synthetically produced DNA molecules depends on the type of the single base pair of which the polymer is built of. While a polaron-like mechanism, relying on the nonlinear coupling between the electron amplitude and radial vibrations of the base pairs, is responsible for long-range and stable ET in $(\mathrm{dG})-(\mathrm{dC})$ DNA polymers, the charge mobility is comparatively weaker in the case of (dA)-(dT) DNA polymers. 
Especially when it comes to designing synthetic molecular wires these findings might be of interest. In fact, recent experiments suggest that ET through DNA molecules proceeds by polaron hopping [31]. Furthermore, our results comply with the findings of these experiments which show also that poly $(\mathrm{dG})$-poly $(\mathrm{dC})$ DNA polymers forms a better conductor than their poly(dA)-poly(dT) counterparts.

\section{Summary}

We have reviewed studies on ET mechanism in DNA relying on the coupling of the charge carrying unit to the vibrational modes of DNA responsible for the formation of polarons and electron-vibron breathers. The steric structure of the double helix of twisted DNA has been described by a network system of coupled oscillators in the context of the base-pair picture considering angular and radial vibrational motions of the base pairs. The coupling between the electronic and vibrational degrees of freedom is established through modulations of the parameters of the electronic system, (described by a tight-binding system) caused by structural changes of the DNA molecule.

We have focused interest on the initiation of long-range and stable polaron and breather motion along the DNA structure when static and/or structural disorder inherent to any real DNA molecule is taken into account (intrinsic static parametrical and/or structural disorder reflecting structural imperfections and the impact of ambient solvent coordinates). As a first step we have constructed exact stationary localized solutions (polarons) with the help of a nonlinear map approach. Motion of the polaron is then activated through a kick mechanism utilizing the pinning mode.

We have shown that directed motion prevails when the electron-vibration system is subjected to randomness. Even for a moderate amount of parametrical and structural disorder the polaron compounds remain in localized shapes and support directed, long-ranged coherent electron transfer along strands of the double helix. Regarding the vibrational degrees of freedom of the DNA we have observed that the electron is accompanied by a vibrational breather in the radial variable causing local and temporal deformations of the traversed region of the double helix. Hence, efficient charge transport in DNA proceeds on the condition that the double helix undergoes structural changes which is exemplary for the interplay of structure and function in flexible and adaptive biomolecules. Conclusively, DNA molecules might be useful for the design of functional nanowires as constituents of molecular nanoelectronical devices. 


\section{Acknowledgements}

JFRA acknowledges funding by the Ministerio de Educación y Ciencia, Spain, project FIS2004-01183.

\section{References}

[1] M.D. Purugganan et al., Science 247 (1988) 6548 ; D.N. Beratan et al., Science 258 (1997) 1740; C.J. Murphy et al., Science 262 (1993) 1025; M.R. Arkin et al.., ibid 273 (1996) 475; P.J. Dandliker, R.E. Holmlin and J.K. Barton, ibid 257 (1997) 1465.

[2] D.D. Eley and D.I. Spivey, Trans. Faraday Soc. 58 (1962) 411.

[3] D.B. Hall, R.E. Holmkin and J.K. Barton, Nature 382 (1996) 731.

[4] D.B. Hall and J.K. Barton, J. Am. Chem. Soc. 119 (1997) 5045.

[5] M.R. Arkin, E.D.A. Stemp, S.C. Pulver and J.K. Barton, Chem. Biol. 4 (1997) 369.

[6] Y. Okahata, T. Kobayashi, K. Tanaka, and M. Shimomura, J. Am. Chem. Soc. 120 (1998) 6165.

[7] E. Meggers, M.E. Michel-Beyerle and B. Giese, J. Am. Chem. Soc. 120 (1998) 12950.

[8] H.-W. Fink and C. Schönenberger, Nature 398 (1999) 407.

[9] P. Tran, B. Alavi and G. Gruner, Phys. Rev. Lett. 85 (2000) 1564.

[10] F.D. Lewis et al., Science 277 (1997) 673.

[11] C.A. Mirkin, R.L. Letsinger R.C. Mucic and J.J. Storhoff, Nature 382 (1996) 607; A.A. Voityuk, N. Rösch, M. Bixon, and J. Jortner, J. Phys. Chem. 104 (2000) 9740; F.C. Grozema, Y.A. Berlin and L.D.A. Siebbels, J. Am. Chem. Soc. 122 (2000) 10903; Y.A. Berlin, A.L. Burin and M.A. Ratner, J. Am. Chem. Soc. 123 (2001) 260.

[12] M. Ratner, Nature 397 (1999) 480.

[13] B. Giese, J. Amaudrut, A.K. Köhler, M. Spormann, and S. Wessely, Nature 412 (2001) 318 and references therein.

[14] A.Yu. Kasumov, M. Kociak, S. Gueron, B. Reulet, V.T. Volkov, D.V. Klinov and H. Bouchiat, Science 291 (2001) 280.

[15] E. Braun, Y. Eichen, U. Sivan and G. Ben-Yoseph, Nature 391 (1998) 775.

[16] D. Porath, A. Bezryadin, S. de Vries and C. Dekker, Nature 403 (2000) 635. 
[17] P.J. de Pablo, F. Moreno-Herrero, J. Colchero, J. Gómez Herrero, P. Herrero, A.M. Baró, P. Ordejón, J.M. Soler and E. Artacho, Phys. Rev. Lett. 85 (2000) 4992.

[18] J. Jortner, M. Bixon, T. Langenbacher and M.E. Michel-Beyerle, Proc. Nat. Acad. Sci. USA 95 (1998) 12759.

[19] Y.-J. Ye, R.-S. Chen, A. Martinez, P. Otto and J. Ladik, Sol. Stat. Comm. 112 (1999) 139.

[20] R. Bruinsma, G. Grüner, M.R. D'Orsogna and J. Rudnick, Phys. Rev. Lett. 85 (2000) 4393.

[21] D.M. Basko and E.M. Conwell, Phys. Rev. E 65 (2002) 061902.

[22] Z.G. Yu and Xueyu Song, Phys. Rev. Lett. 86 (2001) 6018.

[23] M. Hjort and S. Stafström, Phys. Rev. Lett. 87(2001) 228101-1.

[24] D. Ly et al, J. Am. Chem. Soc. 118 (1996) 8747.

[25] J. Jortner, Proc. Nat. Acad. Sci. USA, 95 (1998) 12759.

[26] E. Conwell and S.V. Rakhmanova, Proc. Nat. Acad. Sci. USA, 97 (2000) 4556; S.V. Rakhmanova and E.M. Conwell, J. Phys. Chem. B 105 (2001) 2056.

[27] D. Ly, L. Sanii and G.B. Schuster, J. Am. Chem. Soc., 121 (1999) 9400.

[28] S. Komineas, G. Kalosakas, and A.R. Bishop, Phys. Rev. E 65 (2002) 061905.

[29] Z. Hermon, S. Caspi and E. Ben-Jacob, Europhys. Lett. 43 (1998) 482.

[30] P. Carpena, P. Bernaola-Galvan, P.Ch. Ivanov, and H.E. Stanley, Nature 418 (2002) 955.

[31] L. Cai, H. Tabata, and T. Kawai, Appl. Phys. Lett. 77 (2000) 3105; K.-H. Yoo, D.H. Ha, J.-O. Lee, J.W. Park, Jinhee Kim, J.J. Kim, H.-Y. Lee, T. Kawai, and Han Yong Choi, Phys. Rev. Lett. 87 (2001) 198102; H.-Y. Lee, H. Tanaka, Y. Otsuka, K.-H. Yoo, J.-O Lee, and T. Kawai, Appl. Phys. Lett. 80 (2002) 1670.

[32] M. Peyrard and A.R. Bishop, Phys. Rev. Lett. 62 (1989) 2755.

[33] L.V. Yakushevich, Quart. Rev. Biophys. 26 (1993) 201.

[34] M. Barbi, S. Cocco and M. Peyrard, Phys. Lett. A 253 (1999) 358.

[35] L. Stryer, Biochemistry, Freeman, New York, 1995.

[36] S. Cocco and R. Monasson, J. Chem. Phys. 112 (2000) 10017.

[37] T.D. Holstein, Ann. Phys. NY 8 (1959) 325.

[38] P.W. Anderson, Phys. Rev. 109 (1958) 1492.

[39] N.F. Mott, Adv. Phys. 10 (1961) 107. 
[40] D. Hennig, J.F.R. Archilla and J. Agarwal, Physica D 180 (2003) 256.

[41] F. Palmero, J.F.R. Archilla, D Hennig, and F.R. Romero, N. J. Phys. 6, 13.1 (2004).

[42] D. Hennig, Eur. Phys. J. B 30 (2002) 211.

[43] G. Kalosakas, S. Aubry and G.P. Tsironis, Phys. Rev. B 58 (1998) 3094.

[44] N.K. Voulgarakis and G.P. Tsironis, Phys. Rev. B 63 (2001) 14302.

[45] M. Peyrard and M.D. Kruskal, Physica D 14 (1984) 88.

[46] S. Flach and C.R. Willis, Phys. Rep. 295 (1998) 181.

[47] D. Chen, S. Aubry, and G.P. Tsironis, Phys. Rev. Lett. 77 (1996) 4776.

[48] Y.-J. Ye and J. Ladik, Int. J. Quant. Chem. 52 (1994) 491.

[49] W. Saenger Principles of Nucleic Acid Structure, Springer Verlag, New York, 1984.

[50] G. Brunaud, F. Castet, A. Fritsch, M. Kreissler, and L. Ducasse, J. Phys. Chem. B 105 (2001) 12665.

[51] G. Cuniberti, L. Craco, D. Porath, and C. Dekker, Phys. Rev. B 65 (2002) $241314(\mathrm{R})$.

[52] J.J.P. Stewart, J. Comp. Chem. 10 (1989) 209.

[53] E.B. Starikov, Phys. Chem. Chem. Phys. 4 (2002) 4523.

[54] D. Hennig, E.B. Starikov, J.F.R. Archilla and F. Palmero, J. Biol. Phys. 30 (2004) 227.

[55] A. Fortunelli and A. Painelli, 1997,Phys. Rev. B 55 (1997) 16088.

[56] E.S. Chen and E.C.M. Chen, Biochem.Biophys.Res.Comm. 289 (2001) 421.

[57] M. Ibanes, J.M. Sancho, and G.P. Tsironis, Phys. Rev. E 65 (2002) 041902.

[58] Y. Dakhnovskii, D.G. Evans, H.J. Kim and R.D Coalson, J. Chem. Phys. 103 (1995) 5459.

[59] I.A. Goychuk, E.G. Petrov, and V. May, Chem. Phys. Lett. 253 (1996) 428. 106 (1997) 4522. 